\title{
Cancer cachexia is regulated by selective targeting of skeletal muscle gene products
}

\author{
Swarnali Acharyya, ${ }^{1,2,3}$ Katherine J. Ladner, ${ }^{1}$ Lori L. Nelsen, ${ }^{1}$ Jeffrey Damrauer, ${ }^{1}$ Peter J. Reiser, ${ }^{4}$ \\ Steven Swoap, ${ }^{5}$ and Denis C. Guttridge $1,2,3,6$
}

\begin{abstract}
1Division of Human Cancer Genetics, ${ }^{2}$ Department of Molecular Virology, Immunology and Medical Genetics, ${ }^{3}$ ntegrated Biomedical Graduate Program, and ${ }^{4}$ Department of Oral Biology, The Ohio State University, Columbus, Ohio, USA. ${ }^{5}$ Department of Biology, Williams College, Williamstown, Massachusetts, USA. ${ }^{6}$ The Arthur G. James Comprehensive Cancer Center, The Ohio State University, Columbus, Ohio, USA.
\end{abstract}

\begin{abstract}
Cachexia is a syndrome characterized by wasting of skeletal muscle and contributes to nearly one-third of all cancer deaths. Cytokines and tumor factors mediate wasting by suppressing muscle gene products, but exactly which products are targeted by these cachectic factors is not well understood. Because of their functional relevance to muscle architecture, such targets are presumed to represent myofibrillar proteins, but whether these proteins are regulated in a general or a selective manner is also unclear. Here we demonstrate, using in vitro and in vivo models of muscle wasting, that cachectic factors are remarkably selective in targeting myosin heavy chain. In myotubes and mouse muscles, TNF- $\alpha$ plus IFN- $\gamma$ strongly reduced myosin expression through an RNA-dependent mechanism. Likewise, colon-26 tumors in mice caused the selective reduction of this myofibrillar protein, and this reduction correlated with wasting. Under these conditions, however, loss of myosin was associated with the ubiquitin-dependent proteasome pathway, which suggests that mechanisms used to regulate the expression of muscle proteins may be cachectic factor specific. These results shed new light on cancer cachexia by revealing that wasting does not result from a general downregulation of muscle proteins but rather is highly selective as to which proteins are targeted during the wasting state.
\end{abstract}

\section{Introduction}

Cachexia is a wasting condition that manifests itself in several lifethreatening diseases, including cancer, congestive heart failure, AIDS, and sepsis $(1,2)$. Often misdiagnosed as simply a condition of weight loss, cachexia actually is a highly complex metabolic disorder involving features of anorexia, anemia, lipolysis, activation of acute phase response, and insulin resistance. In addition, patients exhibit a significant loss of lean body mass, resulting from the wasting of skeletal muscle (3). Unlike starvation, which depletes fat stores from adipose tissue while conserving protein from skeletal muscle, in cachexia neither fat nor protein is spared (4). This may explain why nutritional supplements alone promote weight gain due to the replenishment of fat but are mostly ineffective in restoring skeletal muscle protein content and reversing whole-body wasting (5). The reduction in muscle protein culminates in conditions of asthenia, immobility, and cardiac or respiratory failure (1). The cachectic state is particularly problematic in cancer, typifying poor prognosis and often lowering responses to chemotherapy and radiation treatment (3). More than half of cancer patients suffer from cachexia, and strikingly, nearly a third of mortalities are estimated to result from cachexia rather than the tumor burden (6).

Although the molecular basis of cancer cachexia remains largely unresolved, several key mediators have been identified. One set is proinflammatory cytokines, including TNF- $\alpha$ (7), IL-1 $\beta$ (8), IL-6 (9), and IFN- $\gamma(10)$. These factors are produced by host immune cells in response to the tumor, or by tumor cells themselves. TNF- $\alpha$ in

Nonstandard abbreviations used: $\mathrm{BALB} / \mathrm{c} \times \mathrm{DBA} / 2 \mathrm{~F}_{1}(\mathrm{CD} 2 \mathrm{~F} 1)$; co-immunoprecipitation (co-IP); colon-26 (C-26); differentiated medium (DM); myosin heavy chain (MyHC).

Conflict of interest: The authors have declared that no conflict of interest exists.

Citation for this article: J. Clin. Invest. 114:370-378 (2004).

doi:10.1172/JCI200420174. particular (formerly named cachectin and from here on referred to as TNF) has been found to be elevated in the circulation of cancer patients (11-15) and is thought to contribute to multiple aspects of cachexia (16). For example, TNF potently stimulates fat degeneration $(17,18)$ and plays a key role in tumor-induced muscle wasting (19-24). With regard to muscle wasting, however, studies using predifferentiated myofiber cultures or skeletal muscle explants showed that TNF, even at high concentrations, was not sufficient to promote protein loss $(25,26)$. Such results prompted the notion that factors in addition to TNF are required for muscle wasting (27). This claim is supported by observations of protein loss resulting from increases in the pro-cachectic cytokines IL- 6 , IL- $1 \beta$, and IFN- $\gamma$, from an increase in the tumor-producing factor proteolysis-inducing factor (PIF), and from a reduction in the activity of anti-cachectic cytokines like IL-15 (28-30). It is thus likely that wasting is regulated by multiple factors originating from both tumor and host immune cells.

Cachectic factors are thought to regulate muscle wasting by several mechanisms. One is reduction of protein synthesis, through either the inhibition of amino acid uptake or the suppression of RNA. Indeed, in some tumor models total muscle RNA can decrease as much as $40 \%$ compared with that in control animals $(31,32)$. Wasting may also occur through protein breakdown regulated by the lysosomal, the calpain, or the ATP-dependent ubiquitin/proteasome pathway (33). Of these pathways, the ubiquitin/proteasome system is by far the most studied (34). Both inflammatory cytokines and tumor-secreted factors are known to stimulate the expression of ubiquitin and various subunits of the $26 \mathrm{~S}$ proteasome (33), and recently, muscle-specific E3 ligases were identified and shown to stimulate muscle atrophy $(35,36)$.

Though several cachectic factors have been identified and the mechanism of their signaling pathways partly elucidated, surprisingly little attention has been paid to the actual muscle gene products that are targeted for downregulation during the wasting state. 
Because of their overall abundance and functional relevance in skeletal muscle architecture, it has been largely presumed that such targets represent myofibrillar proteins that constitute the basic contractile unit of myofibrils (37). But whether there is selectivity as to which myofibrillar protein is targeted for downregulation is not known, nor have the mechanisms that regulate the expression of these proteins been firmly established. To gain insight into this aspect of cachexia, we examined the regulation of the core myofibrillar proteins myosin heavy chain $(\mathrm{MyHC})$, actin, troponin, and tropomyosin in both cell culture and tumor models of muscle wasting. Our results show that cachectic factors display a remarkably high degree of selectivity for the downregulation of $\mathrm{MyHC}$. Results also indicate that, depending on the inducing signal, loss of $\mathrm{MyHC}$ can occur through either an RNA-dependent or a proteasome-dependent process, which raises the possibility that the mechanism of targeted downregulation depends on the specific cachectic factor mediating the wasting state. Based on these findings, we propose that cancer-induced muscle wasting does not result from a general loss of muscle proteins but rather derives from the selective downregulation of key skeletal muscle gene products.

\section{Results}

Proinflammatory cytokines selectively downregulate $M y H C$. Cancer-induced muscle wasting is mediated in large part by signaling pathways from multiple proinflammatory cytokines as well as from tumor-produced catabolic factors $(3,28)$. Because of their abundance in muscle, it has been largely presumed that the activation of these pathways leads to the targeted downregulation of myofibrillar proteins that constitute the sarcomeric unit of the myofibrils, essential for skeletal muscle function. But which of these muscle gene products serve as bona fide targets of cachectic factors is not clear, and the mechanisms that regulate the levels of these proteins in a wasting state are not well understood. To gain insight into this aspect of cachexia, we initiated this study by examining the effects of proinflammatory cytokines on the expression of myofibrillar proteins in predifferentiated C2C12 cultures. Previous work using this culture system demonstrated that TNF in combination with IFN- $\gamma$ (from here on referred to as IFN) caused a pronounced reduction of $\mathrm{MyHC}$, without affecting the viability of the myotubes (25, 38). To address the specificity of this regulation, $\mathrm{C} 2 \mathrm{C} 12$ myotubes were treated and the expression of other core myofibrillar proteins was examined.

Interestingly, in comparison with $\mathrm{MyHC}$, whose expression decreased nearly fivefold in treated myotubes, no appreciable quantitative loss occurred with any of the other core myofibrillar proteins troponin $\mathrm{T}$, tropomyosin ( $\alpha$ and $\beta$ forms), or $\alpha$-sarcomeric actin (Figure $1, \mathrm{~A}-\mathrm{C}$ ). In addition, TNF/IFN treatment had no effect on the expression of another myofibrillar protein, actinin, nor was any difference detected in the levels of myosin light chain; this suggests that regulation of $\mathrm{MyHC}$ by TNF/ IFN signaling is not a general phenomenon related to thick filament proteins (Figure 1C). A similar level of $\mathrm{MyHC}$ repression was observed with a second anti-

\section{Figure 1}

myosin antibody, demonstrating that this regulation is not antiserum-dependent (Figure 1D). Importantly, primary myotubes treated with TNF/IFN showed a similar downregulation of $\mathrm{MyHC}$, demonstrating that this phenotype was not specific to immortalized C2C12 cells (Figure 1E). Thus, in cultured myotubes MyHC appears to be a selective target of the TNF/IFN signaling pathway.
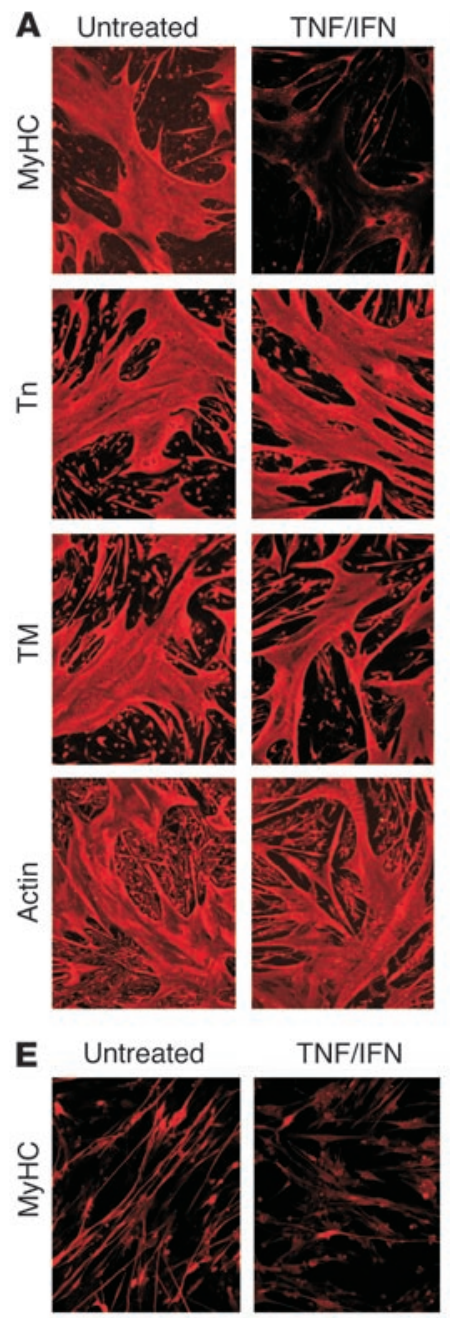

TNF/IFN
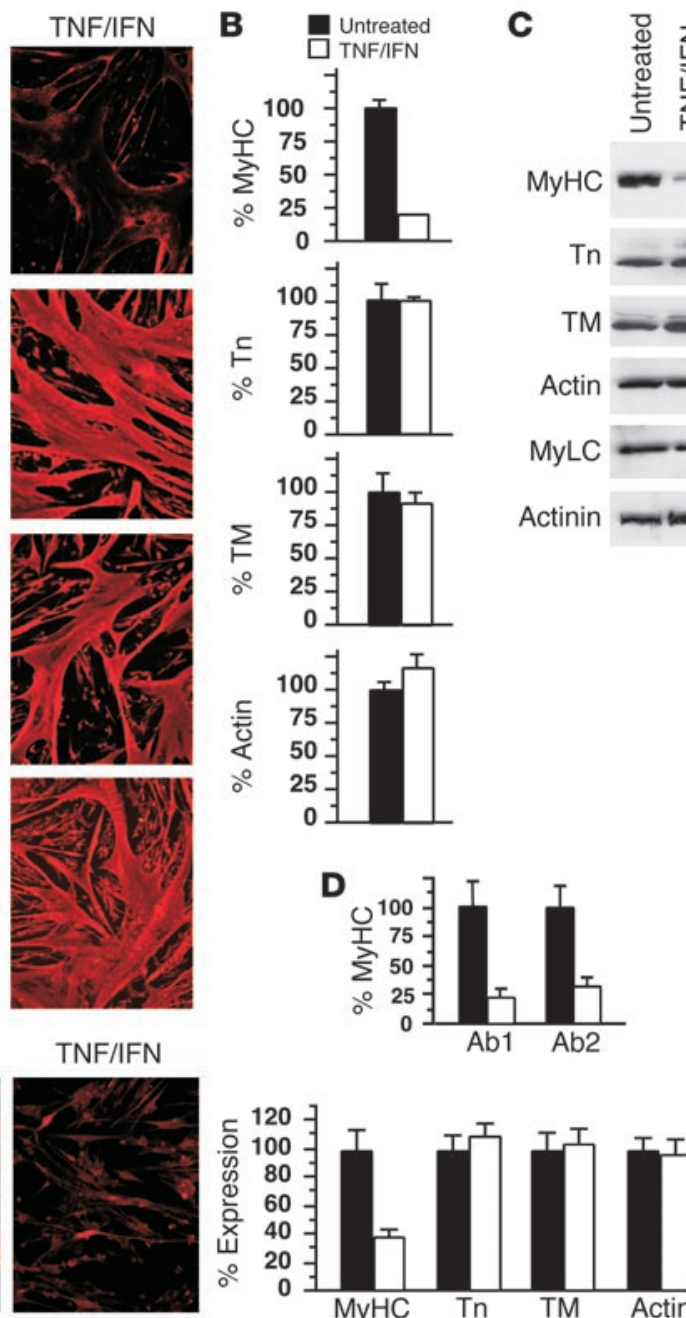

$\mathrm{MyHC}$
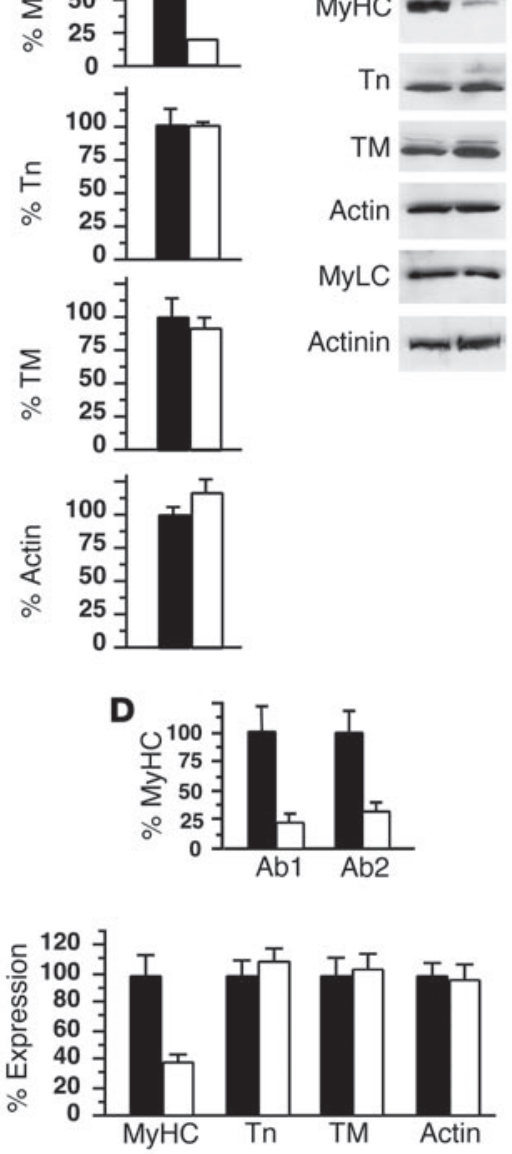

MyHC is selectively targeted by TNF/IFN signaling. C2C12 myoblasts were differentiated in DM for 3 days and subsequently switched to medium alone or containing TNF (10 ng/ml) and IFN (100 U/ml) for 48 hours. (A) Immunofluorescence to detect expression of myofibrillar proteins (MyHC, fast twitch; troponin T [Tn]; tropomyosin, $\alpha$ and $\beta$ [TM]; actin, $\alpha$ skeletal). Images are shown at $\times 20$ magnification. (B) Immunostained expression levels of myofibrillar proteins were quantitated using Zeiss AxioVision software (Carl Zeiss Inc., Thornwood, New York, USA). The data were calculated from a minimum of ten randomly chosen fields of cells at $\times 4$ magnification. (C) Whole-cell extracts were prepared from untreated or TNF/ IFN-treated myocytes, and $50 \mu \mathrm{g}$ total protein was used in Western analyses to probe for myofibrillar proteins (troponin; $\alpha$ - and $\beta$-tropomyosin; MyLC, myosin light chain). (D) Immunofluorescence of untreated (black bars) or TNF/IFN-treated (hatched bars) C2C12 myotubes, probing for MyHC (Ab1, MyHC antibody MY-32; $\mathrm{Ab2}$, MyHC antibody MF20). (E) Primary myotubes were either untreated (black bars) or treated with TNF and IFN (hatched bars) for 48 hours. Cells were stained by immunofluorescence for myofibrillar proteins, and the level of protein expression was quantitated by digital capture and AxioVision software. For histograms, each bar represents mean \pm SEM from three independent experiments. 
A

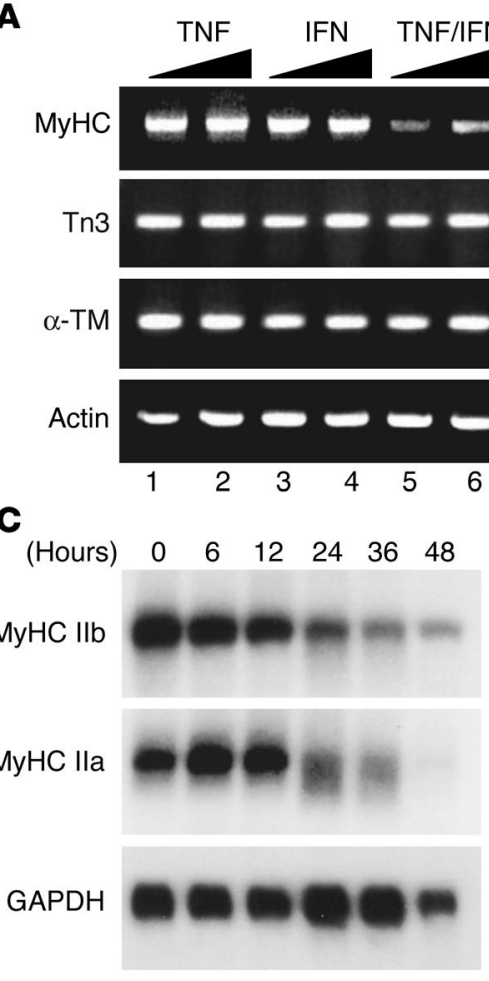

B

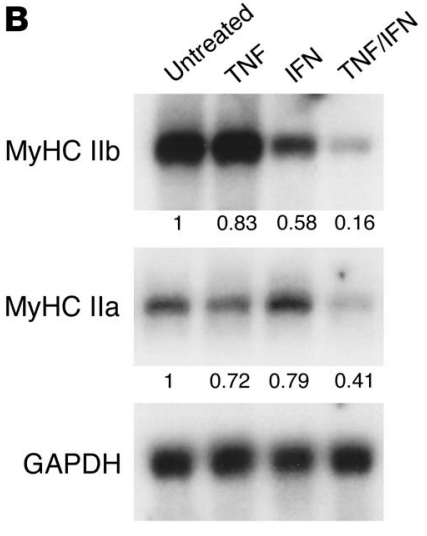

D

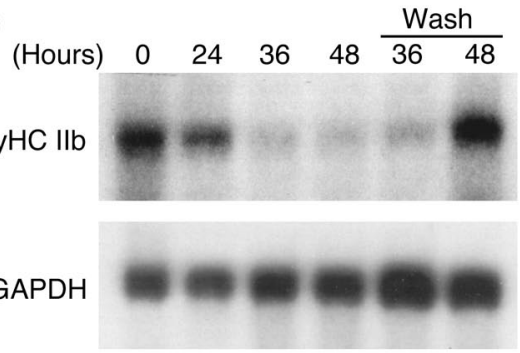

\section{Figure 2}

Chronic TNF/IFN signaling is required to induce the temporal downregulation of MyHC mRNA. (A) Myotubes were treated with DM containing increasing doses of TNF ( $5 \mathrm{ng} / \mathrm{ml}$, lane $1 ; 10 \mathrm{ng} / \mathrm{ml}$, lane 2$)$, IFN (50 U/ml, lane 3; $100 \mathrm{U} / \mathrm{ml}$, lane 4$)$, or TNF plus IFN $(5 \mathrm{ng} / \mathrm{ml}+50 \mathrm{U} / \mathrm{ml}$, lane $5 ; 10 \mathrm{ng} / \mathrm{ml}+100 \mathrm{U} / \mathrm{ml}$, lane 6). After 48 hours, RNA was prepared and RT-PCR was performed (MyHC Ilb; Tn3, troponin T3; $\alpha$-TM, $\alpha$-tropomyosin; $\alpha$-actin). (B) Myotubes were either untreated or treated with TNF $(5 \mathrm{ng} / \mathrm{ml})$, IFN (50 U/ml), or TNF plus IFN $(5 \mathrm{ng} / \mathrm{ml}+50 \mathrm{U} / \mathrm{ml})$ for 48 hours. RNA was prepared, and Northern blots probing for MyHC Ila and IIb were performed. GAPDH was used as a loading control. Numbers are densitometric values normalized to GAPDH. (C) Myotubes were treated with TNF/IFN, and at the indicated times RNA was prepared and Northern blots probing for MyHC type Ila and Ilb were performed. (D) Myotubes were treated with TNF/IFN for 24 hours, at which time cells continued to be treated with cytokines or were washed with PBS and switched to DM (Wash). RNA was prepared at the indicated time points, and Northern blots probing for $\mathrm{MyHC} \mathrm{Ilb} \mathrm{were}$ performed. Similar results were obtained from two additional independent experiments.
Regulation of MyHC by TNF/IFN signaling is RNA-dependent. Given that TNF and IFN share biological properties, and that their respective signaling pathways often synergize to regulate gene expression (39), we asked whether MyHC regulation occurred at the RNA level. RT-PCR results showed that increasing concentrations of TNF or IFN alone had minimal effects on the expression of $\mathrm{MyHC}$. In contrast, addition of both cytokines reduced $\mathrm{MyHC}$ mRNA in cooperative fashion (Figure 2A). Consistent with protein data in Figure 1, expression of troponin, tropomyosin, and actin genes were unchanged by cytokine treatment. Northern analysis confirmed these results and further estimated that TNF/IFN treatment caused an approximately sixfold reduction in $\mathrm{MyHC}$ expression compared with that in control myotubes (Figure 2B), a value similar to that determined at the protein level (Figure 1B). Northern data also revealed that this regulation was not specific to a single myosin isoform, since types IIa and IIb were both regulated by cytokine signaling.

Next the temporal regulation of $\mathrm{MyHC}$ was examined. Reduction in MyHC IIb RNA occurred as early as 6 hours after TNF/IFN treatment, and levels continued to progressively decline between 12 and 48 hours (Figure 2C). Regulation of MyHC IIa was also observed, though the kinetics for this isoform was slower than that for IIb. As predicted, the decrease in MyHC RNA was also closely followed by a similar reduction in myosin protein (Supplemental Figure 1; supplemental material available at http://www.jci.org/cgi/content/full/114/3/370/DC1). To determine whether this regulation was reversible, $\mathrm{MyHC}$ was compared in myotubes either persistently treated with cytokines or washed free of the factors after an initial 24-hour exposure. Compared with the downregulated levels of MyHC mRNA in continually treated myotubes, MyHC mRNA levels were restored in the absence of cytokine stimuli (Figure 2D). Together these data indicate that MyHC selectivity is mediated at the RNA level and regulated in a temporal manner that requires persistent TNF/IFN signaling.

The rapid rate at which TNF/IFN treatment suppressed MyHC IIb RNA expression suggested that this regulation occurred at the transcriptional level. To investigate this possibility, C2C12 myoblasts were transfected with a reporter construct containing the MyHC IIb promoter (40). As expected, promoter activity significantly increased in differentiating $\mathrm{C} 2 \mathrm{C} 12$ myocytes, confirming the functionality of this reporter system (Figure 3A). Next, similar transfections were performed with control and TNF/IFN-treated myotubes. Results showed that MyHC IIb promoter activity was significantly reduced by TNF/IFN treatment in a time-dependent manner (Figure 3B), an effect similar to the temporal decrease in MyHC mRNA (Figure 2C).

Since transcriptional activation of MyHC IIb is known to be under tight control of the myocyte regulatory factor MyoD (41), we speculated that repression of $\mathrm{MyHC}$ IIb transcription could result from the cytokine-mediated inhibition of this transcription factor. In support of this hypothesis, TNF/IFN caused a dramatic loss of MyoD mRNA in C2C12 myotubes (Figure 3C). In addition, exogenous expression of $\mathrm{MyoD}$ rescued $\mathrm{MyHC}$ transcription in the presence of the two cytokines (Figure 3D). This regulation was specific to MyoD, since similar expression of a mutant form of this transcription factor (42) was unable to restore promoter activity. Taken together, these data suggest that one mechanism by which TNF/IFN signaling can repress $\mathrm{MyHC}$ is at the transcriptional level, possibly resulting from the cytokine-mediated inhibition of MyoD synthesis.

To address whether other mechanisms may contribute to the regulation of $\mathrm{MyHC}$, we tested the incorporation rate of $\left[{ }^{35} \mathrm{~S}\right]$-methionine into myosin prior to the major decline of myosin RNA that occurred in response to TNF and IFN. Results from this analysis revealed little difference in incorporation rates, indi- 

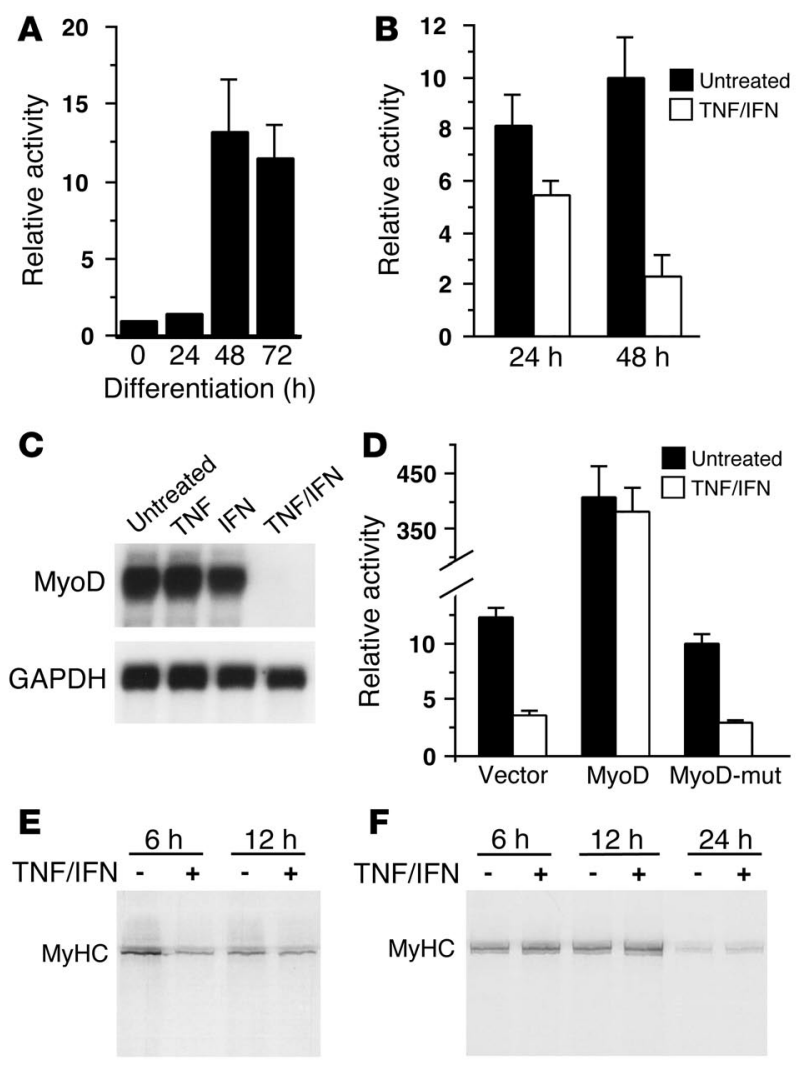

cating that TNF/IFN treatment does not affect the synthesis of MyHC (Figure 3E). $\left[{ }^{35} \mathrm{~S}\right]$ pulse chase analysis also revealed that TNF/IFN treatment had little effect on the rate of MyHC turnover (Figure 3F). Although an effect on MyHC protein cannot be completely ruled out, the data demonstrate that selective targeting of MyHC by TNF/IFN signaling occurs predominantly through an RNA-dependent mechanism.

$M y H C$ is selectively targeted by TNF/IFN signaling in vivo. To determine whether a similar regulation by TNF/IFN occurred in vivo, $\mathrm{CHO}$ cells stably expressing the secreted forms of TNF and IFN were utilized $(18,25)$. Vector control and cytokine-expressing $\mathrm{CHO}$ cells were thus injected intramuscularly in mice, and the expression of myofibrillar gene products was subsequently examined at 6 and 12 days. While the persistent expression of cytokines in vivo led to the reduction of $\mathrm{MyHC}$ protein, no significant decrease was observed for other core myofibrillar proteins (Figure 4A). The decrease in $\mathrm{MyHC}$ was also associated with a concomitant reduction in its mRNA (Figure 4B), while no such effects were observed with other myofibrillar genes. These results thus confirm that among the core myofibrillar proteins, $\mathrm{MyHC}$ is a selected target of TNF/IFN signaling.

\section{Figure 4}

Expression of TNF and IFN in vivo causes the selective downregulation of MyHC. Saline used as a control $(100 \mu \mathrm{l})$ or $\mathrm{CHO}$ cells expressing TNF and IFN $\left(1 \times 10^{7}\right.$ cells per $\left.0.1 \mathrm{ml}\right)$ were injected into gastrocnemius muscles of nude mice ( $n=4$ per group). At 6 and 12 days after injection, muscles were harvested, divided into equal sections, and homogenized to prepare either total protein or RNA. Core myofibrillar gene products were examined by Western blotting $(\mathbf{A})$ or Northern analyses (B) (MyHC Ilb, $\alpha$-tropomyosin, troponin T3, $\alpha$-actin).

\section{Figure 3}

TNF/IFN inhibits MyHC transcription through the concomitant reduction of MyoD. (A) C2C12 cells were transfected in triplicate with a MyHC Ilb-luciferase reporter plasmid as described in Methods. The next day, cells were differentiated and at the indicated times were harvested for luciferase assays. (B) Myoblasts were transfected with MyHC Ilbluciferase and subsequently differentiated for 48 hours, at which time cells were left untreated or treated with TNF/IFN. At the indicated times, cells were harvested and luciferase levels determined. (C) Myotubes were either untreated or treated with TNF $(5 \mathrm{ng} / \mathrm{ml})$, IFN $(50 \mathrm{U} / \mathrm{ml})$, or TNF plus IFN for 48 hours. Northern blots probing for MyoD were performed (25), and GAPDH was used as a loading control. (D) Myoblasts were transfected under conditions similar to those described in $\mathbf{B}$ with a MyHC Ilb-luciferase reporter along with empty vector or MyoD wildtype or mutant (mut) expression plasmids (50 ng each). Cells were differentiated for 48 hours and subsequently left untreated or treated with TNF and IFN for an additional 24 hours, at which time cell extracts were prepared and luciferase activities determined. (E) Myotubes were either untreated or treated with TNF and IFN in methionine/cysteine-free DM, then pulsed for 1 hour with [ $\left.{ }^{35} \mathrm{~S}\right]$-methionine/cysteine prior to collection of cells. Labeled $\mathrm{MyHC}$ was detected by immunoprecipitation. (F) Myotubes were pulsed with DM containing [ $\left.{ }^{35} \mathrm{~S}\right]-$ methionine/cysteine, then chased with fresh DM with or without TNF/IFN. For histograms, the data are representative of experiments performed a minimum of three times, plotted as mean \pm SD.

$M y H C$ is a selective target in colon-26 cancer cachexia. In the final part of this study, we attempted to address whether the selective regulation of MyHC demonstrated by TNF/IFN signaling could be extended to an established cancer-cachexia model of muscle wasting. For experimental convenience, we chose the colon-26 (C-26) adenocarcinoma model (43-45). Although muscle wasting in this tumor model is largely accepted to be dependent on IL- 6 rather than TNF or IFN $(43,46)$, we reasoned that if $\mathrm{MyHC}$ were indeed a selective target of myofibrillar proteins in cachexia, this regulation would be maintained irrespective of the cachectic factor inducing the wasting state. To test this hypothesis, C-26 adenocarcinoma tumors were established in mice. Consistent with previous reports (43-45), mice bearing C-26 tumors underwent substantial weight loss (Figure 5A) and exhibited other classic features of cachexia, including anorexia, enlarged spleen, and reduced heart size (data not shown). In addition, we observed that fat content was nearly completely depleted following 12 days of tumor inoculum, while loss of muscle mass was only beginning to occur at this same time (Table 1). Muscle weights continued to decline from day 12 onward, which we suggest likely accounted for the persistent reduction in whole-body weights. In addition, like that observed in cancer patients (47), wasting was more pronounced in fast-twitch

A

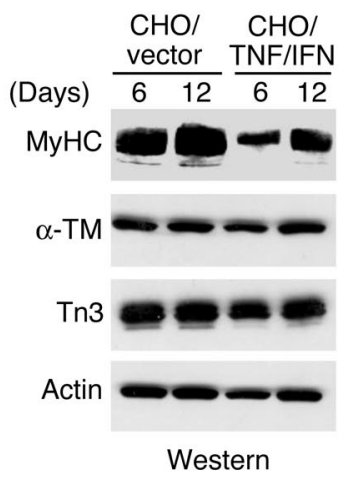

B

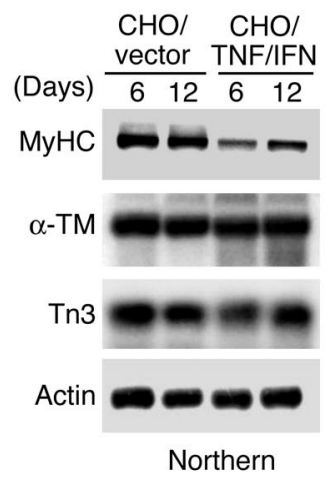


A

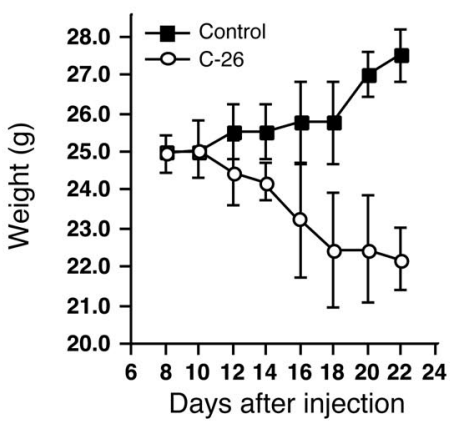

B

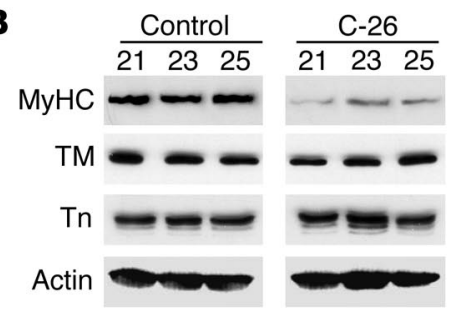

C

MyHC Ilb $\frac{\text { Control }}{212325} \frac{\text { C-26 }}{212325}$

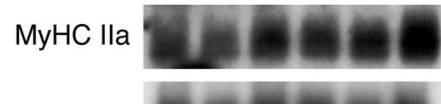

$\alpha-\mathrm{TM}$

Tn3

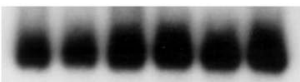

Actin

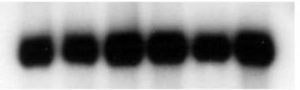

D

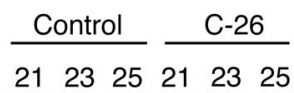

Figure 5

C-26 tumors induce severe muscle wasting associated with a selective loss of MyHC protein. (A) Saline used as control $(100 \mu \mathrm{l})$ or $\mathrm{C}-26$ adenocarcinoma cells $\left(1 \times 10^{6}\right.$ cells per $0.1 \mathrm{ml}$ ) were injected subcutaneously into the right flank of CD2F1 male mice. Body weights of salineor C-26-injected mice ( $n=4$ per group) were recorded every 48 hours from day 8 to day 22 postinjection. (B and C) Mice $(n=6)$ were injected in the right flank with either saline (control) or C-26 cells. At the indicated days after injection, tibialis anterior muscles from the right hind limb were collected and divided into two sections. From one section, tissue homogenates were prepared, and Western blot analyses probing for myofibrillar proteins were performed (B). From the remaining section, total RNA was prepared, and Northern blot analyses were undertaken (C). (D) Extracts of soleus muscles from mice described in $\mathbf{B}$ and $\mathbf{C}$ were prepared and used for Western blot analysis for expression of MyHC type I and $\alpha$-sarcomeric actin.

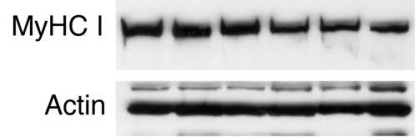

type II-containing muscles, such as tibialis anterior and gastrocnemius, than in slow-twitch type I muscles, such as soleus.

Extracts prepared from control and cachectic tibialis anterior muscles were analyzed for expression of myofibrillar proteins. Results showed that reproducibly lower levels of $\mathrm{MyHC}$ were expressed by cachectic muscles than by non-tumor-bearing control samples (Figure 5B). Similar results were found in gastrocnemius muscles (data not shown). Importantly, in keeping with findings obtained from cytokine-treated myotube cultures and mouse muscles, no detectable changes were observed in the levels of the other myofibrillar proteins. Such results argue strongly that $\mathrm{MyHC}$ is indeed a selective target associated with a wasting state. Although fiber-type switching has been reported to occur in soleus muscles from C-26-bearing mice (48), we were not able to detect such regulation in either tibialis or gastrocnemius muscles (data not shown). In addition, correlating with the lack of appreciable wasting, only a slight difference in $\mathrm{MyHC}$ type I expression was observed in soleus muscles from cachectic mice compared with control animals (Figure 5C).

Next, Northern analyses were performed to determine the mechanism of $\mathrm{MyHC}$ regulation in $\mathrm{C}-26$ cachectic muscles. Interestingly, unlike the decreased levels of MyHC mRNA detected in TNF/ IFN-treated cells or muscles, in this model MyHC mRNA levels were unchanged (Figure 5D). These results indicate that although different causative factors of cachexia maintain an ability to selectively regulate $\mathrm{MyHC}$ expression, this regulation can apparently occur through distinct mechanisms.

Evidence that C-26 tumors target MyHC through the ubiquitin/proteasome pathway. The ubiquitin ligase-dependent proteasome pathway has been identified as a major cellular mechanism that regulates skeletal muscle wasting in cancer and other disease states $(35,36,49)$. Given that no change in MyHC mRNA expression was detected in C-26 cachectic muscles, we asked whether decreases observed at the protein level could be mediated through such a degradation process. In keeping with reports in other tumor models of wasting (50-52), we found that expression of ubiquitin, and of the musclespecific E3 ubiquitin ligase genes $M u R F 1$ and atrogin-1/MAFbx, was visibly increased in muscles with C-26-induced cachexia com- pared with control muscles (Figure 6A). Based on these results, we reasoned that the activation of the ubiquitin/proteasome system would also lead to an increase in the ubiquitinated state of $\mathrm{MyHC}$. To test this, ubiquitin co-immunoprecipitation (co-IP) analyses were performed with extracts prepared from control and C-26 tumor-bearing mice. Results showed that the mature form of $\mathrm{MyHC}$ could readily be immunoprecipitated with ubiquitin; this supports the involvement of this proteolytic pathway in the basal turnover of myosin (Figure 6B). We also detected that levels associated with the mature form of myosin did not substantially differ between tumor and control muscle samples (Figure 6B), which suggests that tumor burden in this case did not enhance the polyubiquitination state of $\mathrm{MyHC}$. Importantly, however, elevated levels of smaller MyHC-reactive fragments were clearly visible in C-26 cachectic muscles, which may represent proteolyzed intermediates of this protein. Furthermore, we observed that in some cachectic muscles taken from mice with late-stage tumors, the ubiquitin antibody precipitated significantly less myosin (Figure 6B, lane 6). Although it is possible that ubiquitin conjugation for $\mathrm{MyHC}$ was defective in these mice, this may also suggest that in these muscles myosin had already undergone substantial degradation.

To validate these findings, similar co-IP reactions were performed with TNF/IFN-treated C2C12 myotubes. Similar to what was observed in muscle extracts, myosin from untreated cells was found in complex with ubiquitin, and as MyHC levels decreased in response to TNF/IFN treatment, progressively less protein was immunoprecipitated with the ubiquitin antibody (Figure 6C). However, unlike in C-26 muscle extracts, less myosin-reactive fragments were detected in treated myotubes. This result, in conjunction with our earlier finding that TNF/IFN did not alter the rate of MyHC turnover (Figure 3), supports the notion that proteolysis is not likely to be a predominant mechanism by which myosin is selectively downregulated in cultured myotubes.

To extend the specificity of these reactions, ubiquitin co-IP was repeated to analyze the association with other core myofibrillar proteins. In comparison with myosin, relatively low levels of the other myofibrillar proteins were found coupled to ubiquitin 
Table 1

Tissue weights from control and tumor-bearing mice

\begin{tabular}{lcccccc}
\hline & \multicolumn{2}{c}{ Day 12 } & \multicolumn{2}{c}{ Day 18 } & \multicolumn{2}{c}{ Day 25 } \\
& Control & C-26 & Control & C-26 & Control & C-26 \\
& & & & & & \\
$\begin{array}{l}\text { Adipose tissue weights } \\
\text { Epididymal fat }\end{array}$ & $285 \pm 22$ & $8 \pm 0$ & $236 \pm 10$ & ND & $279 \pm 14$ & ND \\
$\begin{array}{l}\text { Scapular fat } \\
\text { Skeletal muscle weights }\end{array}$ & $47 \pm 3$ & ND & $63 \pm 3$ & ND & $59 \pm 4$ & ND \\
$\begin{array}{l}\text { Tibialis } \\
\text { Gastrocnemius }\end{array}$ & $49 \pm 9$ & $34 \pm 4$ & $63 \pm 6$ & $29 \pm 7$ & $70 \pm 7$ & $34 \pm 5$ \\
Soleus & $140 \pm 10$ & $115 \pm 5$ & $142 \pm 7$ & $81 \pm 6$ & $145 \pm 13$ & $80 \pm 10$ \\
& $7 \pm 1$ & $8 \pm 1$ & $7 \pm 1$ & $6 \pm 1$ & $10 \pm 1$ & $10 \pm 1$ \\
\hline
\end{tabular}

Saline control or C-26 adenocarcinoma cells were injected subcutaneously into the right flank of CD2F1 mice ( $n=6$ per group). At indicated days, skeletal muscle and adipose tissue weights were recorded in milligrams. Results are represented as mean \pm SEM. Statistical significance was determined using AWilcoxon rank sum test, $P<0.025$, or ${ }^{B}$ Student's $t$ test, $P<0.05$. ND, not detectable.

results demonstrate that selective loss of $\mathrm{MyHC}$, controlled either through an RNAdependent or a protein-dependent mechanism, leads to a concomitant dissociation of myosin-actin interactions.

\section{Discussion}

This study reveals several key findings with regard to muscle wasting in cancer cachexia. The first, and most important, is that pro-cachectic factors do appear to have a high degree of selectivity as to which skeletal muscle gene product is targeted for downregulation. We found that of the myofibrillar proteins examined, $\mathrm{MyHC}$ was pronouncedly downregulated, while no significant changes occurred in the expression levels of tropomyosin, troponin, sarcomeric

in mouse muscles, and in each case no differences in ubiquitin association with these proteins were observed between control and tumor-bearing muscles, or between untreated and TNF/ IFN-treated myotubes (Figure 6, B and C; tropomyosin is used as a representative protein). Taken together, these results imply that C-26-induced muscle wasting is associated with the selective reduction of $\mathrm{MyHC}$ protein, whose regulation is likely to be mediated by ubiquitin-dependent proteolysis.

Finally, to assess the functional significance associated with the loss of $\mathrm{MyHC}$ in muscle wasting, co-IP reactions were performed between myosin and its main binding partner, sarcomeric actin (37). Results showed that myosin-actin complexes were significantly compromised in muscles from tumor-bearing mice compared with control samples (Figure 6D). Consistent with these findings, a severe decrease in myosin-actin association was also detected in C2C12 myotubes treated with TNF and IFN (Figure 6E). These actin, actinin, and myosin light chain. MyHC selectivity was shown in cultured myotubes and mouse muscles treated with TNF/IFN, as well as in mice bearing C-26 tumors. In addition to these cachectic factors, we have extended our analysis by investigating the effects of other known mediators of muscle wasting. Results showed that myotubes treated with TNF and either high concentrations of IL-6 or medium conditioned with G361 melanoma cells also caused the selective downregulation of MyHC (Supplemental Figure 2). Furthermore, selective targeting of $\mathrm{MyHC}$ was also observed in mice bearing G361 melanomas, tumors previously shown to secrete the muscle proteolysis factor proteolysis-inducing factor (PIF) (53). Although other myofibrillar proteins remain to be investigated, these data strongly support that $\mathrm{MyHC}$ is a preferred target of multiple pro-cachectic factors. Given that MyHC accounts for approximately $40 \%$ of myofibrillar protein content in mature muscle (37), and mice lacking this gene display growth and muscle

\section{Figure 6}

C-26 tumors enhance ubiquitin/E3 ligase expression and $\mathrm{MyHC}$ ubiquitination products. Mice were injected in the right flank with either control $(n=3)$ or C-26 cells $(n=3)$, and at days 23-25 after injection, tibialis muscles were isolated and prepared for RNA or protein analyses. (A) Northern blots probing for ubiquitin (Ub), MuRF1, and atrogin-1/MAFbx in muscles from control versus $\mathbf{C}-26$-injected mice. (B and C) Homogenates were prepared from mouse muscles or $\mathrm{C} 2 \mathrm{C} 12$ myotubes, and protein complexes were subsequently immunoprecipitated overnight with an anti-ubiquitin antibody (IP). Immunoprecipitates were fractionated by SDSPAGE, and immunoblot (IB) analyses probing for MyHC (upper panels) or tropomyosin (TM; lower panels) were performed. The Western blot for MyHC in B was purposely overexposed to accentuate differences in MyHC protein products coupled to ubiquitin (bracketed). Asterisks in the tropomyosin Western blot denote nonspecific bands. (D and E) Homogenates described in $\mathbf{B}$ and $\mathbf{C}$ were immunoprecipitated with an $\alpha$-sarcomeric actin antibody (IP), and complexes were fractionated by SDS-PAGE and immunoblotted (IB) for MyHC and $\alpha$-actin.

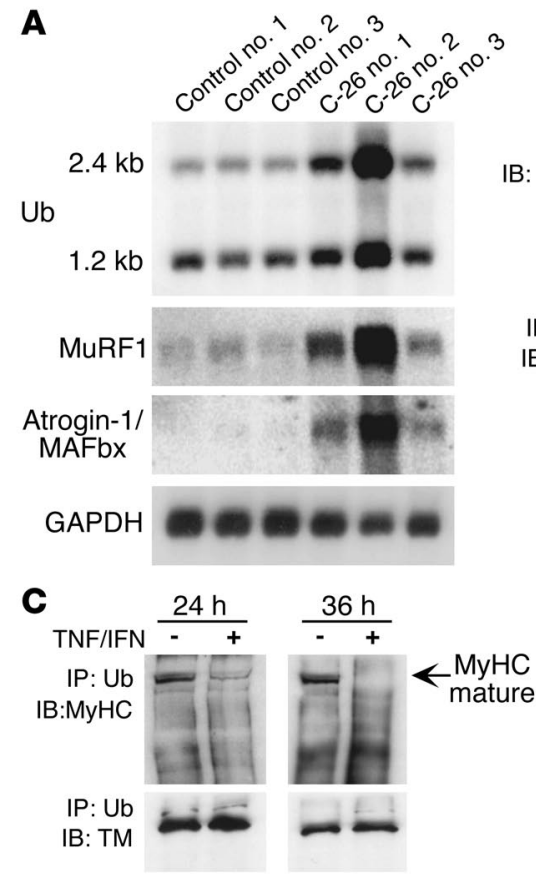


defects (54), it is reasonable to propose that loss of $\mathrm{MyHC}$ would contribute significantly to the reduction in lean mass associated with a cachectic state.

A second finding revealed by this study is that inflammatory cytokines are capable of repressing $\mathrm{MyHC}$ at the RNA level. The data showed that TNF alone was not sufficient to regulate $\mathrm{MyHC}$ mRNA expression (Figure 2), which is consistent with the notion that in vivo TNF functions in concert with other inflammatory cytokines and/or tumor factors to promote muscle wasting (26, $27,55)$. Although our findings demonstrate the dual requirement of TNF and IFN for suppression of $\mathrm{MyHC}$, it should be noted that some reports do maintain the individual role of TNF in muscle wasting $(56,57)$. In addition, administration of TNF in animals has been shown to lead to the reduction of MyHC mRNA (58), although under these conditions the effect may be secondary to the direct activity of the cytokine. Whether circulating levels of TNF or IFN are elevated in cancer patients is controversial, but studies do support their relevance in wasting (11-15). There is also evidence that cytokines may rise as a consequence of the cancer therapy itself. Cachexia-like symptoms have been reported as common side effects of IFN therapy in cancer patients (59), and elevated IFN levels were also reported in patients receiving IL- 2 and IFN- $\beta$ therapy (60). We envision that regulation of myosin at the RNA level may be a contributing mechanism to muscle breakdown in conditions that favor persistent signaling from both TNF and IFN.

Which signaling molecules mediate the cooperative activity of TNF and IFN is not yet known, but there is good reason to suspect

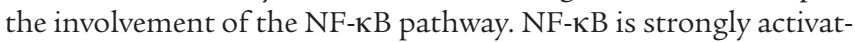
ed by TNF, and recently its activity was shown to be required for cytokine-mediated repression of MyHC protein $(25,56,61)$. NF- $\mathrm{BB}$ was also shown to be required for TNF and IFN to downregulate the expression of MyoD protein in C2C12 myotubes (25). Consistent with these findings, the data presented in this study showed that TNF/IFN could dramatically reduce MyoD mRNA expression, and that rescued expression of MyoD in C2C12 myotubes was sufficient to block TNF/IFN-mediated repression of the MyHC IIb promoter. Given that MyoD binding to the MyHC IIb promoter is required for myosin expression in fast-twitch muscles (41), it is tempting to speculate that cytokine-induced suppression of $\mathrm{MyHC}$ IIb transcription is regulated by NF- $\kappa \mathrm{B}$ through the inhibited synthesis of MyoD. However, it is possible that in addition to $\mathrm{MyoD}$ other myogenic regulators can be targeted by cytokines, leading to the further downregulation of myosin.

Lastly, findings of this study demonstrate that selective targeting of key muscle gene products, such as $\mathrm{MyHC}$, can occur through different regulatory mechanisms. In contrast to TNF/ IFN-treated myotubes, selective targeting of $\mathrm{MyHC}$ in mice bearing C-26 tumors occurred at the protein level. Induction of ubiquitin and E3 ligase genes, in conjunction with the presence of ubiquitin-MyHC conjugates, supports the involvement of the ubiquitin-dependent proteasome system in the regulation of this myofibrillar protein. Thus, these data imply that $\mathrm{MyHC}$ is a preferred substrate of ubiquitin ligases. In vitro, core myofibrillar proteins have been shown to serve as efficient substrates for ubiquitin conjugation, resulting in their degradation by the ATP-dependent 26S proteasome (62). Importantly, the rate of this proteolysis was significantly reduced when core myofibrillar proteins were preassembled into a myofibril complex. This implied that the dissociation of myofibrillar proteins in the contractile apparatus is the rate-limiting step for ubiquitina- tion and subsequent ATP-dependent proteolysis. Consistent with this thinking, sepsis-induced muscle cachexia was reported to be associated with the disruption of sarcomere architecture and the subsequent release of myofilaments, although in this model these effects were largely found to be regulated by a calcium-dependent proteolysis pathway (63). In line with these findings, we predict that C-26 tumor-induced muscle wasting is associated with the disorganization of the myofibrillar network, and that the specificity of $\mathrm{MyHC}$ downregulation in $\mathrm{C}-26$ tumor-bearing mice derives from the partial dissociation of $\mathrm{MyHC}$ from actin and the remaining components of the sarcomeric complex. Upon dissociation of the myofibril, MyHC would thus be susceptible to ubiquitin ligase activity and primed for proteasome-dependent degradation. The absence of actin, troponin, or tropomyosin degradation may signify that these proteins remain bound to each other, thereby minimizing their polyubiquitination. Although our current data demonstrate that myosin and actin association is lost in cachectic muscle (Figure 6), it will be interesting to test whether interactions are compromised between myosin and the other core myofibrillar proteins, as well as the interactions of the core proteins not found to be susceptible to proteolysis.

Based on these current results, we propose that muscle wasting is not a process regulated by the downregulation of a general number of myofibrillar proteins but rather is highly selective as to the targeting of key muscle gene products. Data presented here from different models of cytokine- and tumor-induced cachexia indicate that $\mathrm{MyHC}$ is a selective target irrespective of the pro-cachectic factor. The identification of $\mathrm{MyHC}$ as one of these selective targets may be useful in the design of future cancer-cachexia therapies.

\section{Methods}

Cell culture and materials. Murine C2C12 and primary myoblasts were grown and differentiated in differentiated medium (DM), as previously described (24). Murine C-26 adenocarcinoma cells were cultured with RPMI 1640 (Invitrogen Corp., Carlsbad, California, USA) containing 10\% FBS (HyClone, Logan, Utah, USA). TNF and IFN were purchased from Roche Applied Science (Indianapolis, Indiana, USA). Antibodies, MyHC fast (MY-32), MyHC slow (NOQ7.5.4D), troponin T (JLT-12), sarcomeric tropomyosin (CH1), and $\alpha$-sarcomeric actin (5C5) were obtained from Sigma-Aldrich (St. Louis, Missouri, USA). A MyHC antibody that recognizes all sarcomeric forms of myosin (MF20) was obtained from Developmental Studies Hybridoma Bank (University of Iowa, Iowa City, Iowa, USA). Antibodies for myosin light chain (FL-172) and $\alpha$-actinin (H-300) were obtained from Santa Cruz Biotechnology Inc. (Santa Cruz, California, USA) and ubiquitin from Calbiochem (San Diego, California, USA).

Transfections. Transient transfections were performed in triplicate in $\mathrm{C} 2 \mathrm{C} 12$ cells seeded in 12-well culture dishes. The CMV- $\beta$-galactosidase plasmid was used to standardize for transfection efficiency. Briefly, 0.25 $\mu \mathrm{g}$ of both the MyHC IIb promoter luciferase and CMV- $\beta$-galactosidase plasmids were incubated with SuperFect as recommended by the manufacturer (QIAGEN Inc., Valencia, California, USA). This mixture was subsequently added to the cells with $0.6 \mathrm{ml}$ complete medium for approximately 3 hours. After this time, cells were rinsed with PBS and then incubated with $1 \mathrm{ml}$ complete medium overnight. The following day, cells were induced to differentiate until cell extracts were prepared and luciferase activity was monitored as previously described (24).

Immunofluorescence, Western blot, and co-IP analyses. Myoblasts grown in $35-\mathrm{mm}$ plates were differentiated for a maximum of 3 days and subsequently switched to fresh DM with or without cytokines. After 24 hours, medium was replenished with additional cytokines for $12-24$ hours, after 
which the cells were fixed and stained as previously described (38). For Western analysis, cells were subjected to conditions similar to those for immunofluorescence, and extracts were prepared as previously described (24). For muscle extracts, frozen tibialis anterior or gastrocnemius tissues were homogenized in $1 \mathrm{ml}$ of lysis buffer (1\% Triton X-100, $150 \mathrm{mM}$ $\mathrm{NaCl}, 50 \mathrm{mM}$ Tris- $\mathrm{HCl}$ [pH 7.5], 1 mM EDTA, 1 mM PMSF) using a tissue homogenizer. Immunoblotting was performed by standard techniques. For immunoprecipitation reactions, $250 \mu \mathrm{g}$ of total protein from $\mathrm{C} 2 \mathrm{C} 12$ extracts was used in each reaction. For tissue, tibialis anterior muscles were homogenized in $1 \mathrm{ml}$ of immunoprecipitation lysis buffer $(0.5 \%$ Triton $\mathrm{X}-100,150 \mathrm{mM} \mathrm{NaCl}, 50 \mathrm{mM}$ Tris- $\mathrm{HCl}$ [pH 7.5], $1 \mathrm{mM}$ EDTA, $1 \mathrm{mM}$ PMSF, with standard protease and phosphatase inhibitors), and $500 \mu \mathrm{g}$ of protein was used for each reaction. Extracts were precleared of nonspecific immunoprecipitates with $1 \mu \mathrm{g} \operatorname{IgG}$ for 1 hour at $4^{\circ} \mathrm{C}$. Supernatants were transferred to a fresh tube, and $1 \mu \mathrm{g}$ of antibody was added to the tube and incubated by rocking overnight at $4^{\circ} \mathrm{C}$. After immunoprecipitation, complexes were collected, washed, and resuspended in $30 \mu \mathrm{l}$ in standard loading buffer. Western blots were performed to probe for myofibrillar proteins. Radiolabeled immunoprecipitation reactions were performed in a similar fashion with the following modifications. C2C12 cells were differentiated in DM for 3 days and subsequently treated with or without TNF/IFN in medium lacking methionine and cysteine. One hour prior to cell harvesting, cultures were pulsed with $72 \mu \mathrm{Ci}$ of $\left[{ }^{35} \mathrm{~S}\right]$ EasyTag protein labeling mix (NEN Life Science, Boston, Massachusetts, USA). Alternatively, myotubes were pulsed with $\left[{ }^{35} \mathrm{~S}\right]$ for 1 hour, and then chased with DM with or without TNF/IFN. Immunoprecipitation complexes were resuspended in 30 $\mu \mathrm{l}$ in loading buffer and fractionated by SDS-PAGE. Gels were dried and subsequently exposed to autoradiography for 1-2 days.

Northern blot and RT-PCR analyses. Total RNA was isolated from C2C12 cells or muscle tissues, and Northern blotting was performed as previously described $(24,25)$. For semiquantitative RT-PCR analysis, $2 \mu \mathrm{g}$ of total RNA was used in reactions with Access RT-PCR according to the manufacturer's instructions (Promega Corp., Madison, Wisconsin, USA). Primer pairs for the amplification of myofibrillar gene products were the following: MyHC IIb, 5'-GAATGCCTATGAGGAGTCTCTGG-3' forward, 5'-CTTCTTGGTGTTGATGAGGCTGG-3' reverse; MyHC IIa, 5'-CGTCTGACAGCGAAGAAGCAGG-3' forward, 5'-CTTCTCGGCTTTAT TCCTGGAGG-3' reverse; troponin T3, 5'-GAACCAGAGGAGAAACCAAGACC-3' forward, $5^{\prime}$-GTCATCTTCAGCTCTCCTCTTGG-3' reverse; $\alpha$-tropomyosin, 5'-GCTGAAGCTCGACAAAGA GAACG-3' forward, 5'-CTCGGCTTTCAAT-
GACTTTCATGC-3' reverse; $\alpha$-sarcomeric actin, $5^{\prime}$-GCTGAACGTGAGATTGTGCGCG-3' forward, 5'-GCACGATTGTCGATTGTCGTCC-3' reverse; $M u R F 1$, 5'-GGACGGAAATGCTATGGAGAACC-3' forward, 5'-GATGGCTGTTTCCA CAAGCTTGG-3' reverse; atrogin-1/MAFbx, 5'-CCTCAGCAGTTACTGCAACAAGGA-3' forward, 5'-CATCTTCTTCCAATCCAGCTGCC-3' reverse. The plasmid containing the ubiquitin $\mathrm{CDNA}$ was a generous gift of Y. Xiong (University of North Carolina, Chapel Hill, North Carolina, USA).

Mice and measurement of cachexia. NCR Nu/Nu mice (Taconic, Germantown, New York, USA) or male BALB/c $\times$ DBA $/ 2 \mathrm{~F}_{1}(\mathrm{CD} 2 \mathrm{~F} 1)$ mice (Harlan, Indianapolis, Indiana, USA) were purchased at 6-7 weeks of age, weighing from 23 to $25 \mathrm{~g}$. Animals were housed in the animal facility at the Arthur G. James Comprehensive Cancer Center (The Ohio State University) under conventional conditions with constant temperature and humidity, and fed a standard diet. Treatment of mice was in accordance with the guidelines of the Institutional Animal Care and Use Committee. For in vivo administration of cytokines, $\mathrm{CHO}$ cells expressing either TNF or IFN were injected into gastrocnemius muscles as previously described (25). For tumor administration, cultured C-26 cells were trypsinized, pelleted in complete medium, and subsequently resuspended in PBS at a concentration of $1 \times 10^{7}$ cells $/ \mathrm{ml}$. One hundred microliters of cells were injected subcutaneously into the right flank of CD2F1 mice. Tumor size and body weight were monitored every day starting at day 8 after tumor cell inoculum. Animals were sacrificed at indicated times, and tissues were removed and weighed. Skeletal muscle samples were subsequently snap-frozen in liquid nitrogen and stored at $-70^{\circ} \mathrm{C}$.

Statistical analysis. Analysis was performed between different groups using a two-tailed Student's $t$ test and the Wilcoxon rank sum test.

\section{Acknowledgments}

The authors wish to thank members of the Guttridge laboratory and S. Liyanarachchi for their experimental advice and helpful discussions throughout the course of this study. This work was funded by NIH grants CA097953 and CA098466 and by the V Foundation.

Received for publication October 1, 2003, and accepted in revised form June 3, 2004.

Address correspondence to: Denis C. Guttridge, Division of Human Cancer Genetics, $420 \mathrm{~W}$. Twelfth Avenue, The Ohio State University College of Medicine, Columbus, Ohio 43210, USA. Phone: (614) 6883137; Fax: (614) 688-4006; E-mail: guttridge-1@medctr.osu.edu.
1. Tisdale, M.J. 1997. Biology of cachexia. J. Natl. Cancer Inst. 89:1763-1773.

2. Argiles, J.M., Moore-Carrasco, R., Fuster, G., Busquets, S., and Lopez-Soriano, F.J. 2003. Cancer cachexia: the molecular mechanisms. Int. J. Biochem. Cell Biol. 35:405-409.

3. Tisdale, M.J. 2002. Cachexia in cancer patients. Nat. Rev. Cancer. 2:862-871.

4. Body, J.J. 1999. The syndrome of anorexia-cachexia. Curr. Opin. Oncol. 11:255-260.

5. Evans, W.K., et al. 1985. Limited impact of total parenteral nutrition on nutritional status during treatment for small cell lung cancer. Cancer Res. 45:3347-3353.

6. van Eys, J. 1985. Nutrition and cancer: physiological interrelationships. Annu. Rev. Nutr. 5:435-461.

7. Tracey, K.J., and Cerami, A. 1993. Tumor necrosis factor, other cytokines and disease. Annu. Rev. Cell Biol. 9:317-343.

8. Gelin, J., et al. 1991. Role of endogenous tumor necrosis factor alpha and interleukin 1 for experimental tumor growth and the development of cancer cachexia. Cancer Res. 51:415-421.

9. Barton, B.E. 2001. IL-6-like cytokines and cancer cachexia: consequences of chronic inflammation.
Immunol. Res. 23:41-58.

10. Matthys, P., et al. 1991. Severe cachexia in mice inoculated with interferon-gamma-producing tumor cells. Int. J. Cancer. 49:77-82.

11. Aderka, D., et al. 1985. Cachectin/tumour-necrosis-factor production by cancer patients. Lancet. 2:1190.

12. Balkwill, F., et al. 1987. Evidence for tumour necrosis factor/cachectin production in cancer. Lancet. 2:1229-1232.

13. Nakashima, J., et al. 1998. Association between tumor necrosis factor in serum and cachexia in patients with prostate cancer. Clin. Cancer Res. 4:1743-1748.

14. Karayiannakis, A.J., et al. 2001. Serum levels of tumor necrosis factor-alpha and nutritional status in pancreatic cancer patients. Anticancer Res. 21:1355-1358.

15. Aleman, M.R., et al. 2002. Leptin role in advanced lung cancer. A mediator of the acute phase response or a marker of the status of nutrition? Cytokine. 19:21-26.

16. Argiles, J.M., et al. 2000. The divergent effects of tumour necrosis factor-alpha on skeletal muscle: implications in wasting. Eur. Cytokine Netw.
11:552-559.

17. Ruan, H., Hacohen, N., Golub, T.R., Van Parijs, L., and Lodish, H.F. 2002. Tumor necrosis factor-alpha suppresses adipocyte-specific genes and activates expression of preadipocyte genes in 3T3L1 adipocytes: nuclear factor-kappaB activation by TNF-alpha is obligatory. Diabetes. 51:1319-1336.

18. Oliff, A., et al. 1987. Tumors secreting human $\mathrm{TNF} /$ cachectin induce cachexia in mice. Cell. 50:555-563.

19. Tracey, K.J., et al. 1988. Cachectin/tumor necrosis factor induces cachexia, anemia, and inflammation. J. Exp. Med. 167:1211-1227.

20. Costelli, P., et al. 1993. Tumor necrosis factor-alpha mediates changes in tissue protein turnover in a rat cancer cachexia model. J. Clin. Invest. 92:2783-2789.

21. Llovera, M., et al. 1998. Role of TNF receptor 1 in protein turnover during cancer cachexia using gene knockout mice. Mol. Cell. Endocrinol. 142:183-189.

22. Layne, M.D., and Farmer, S.R. 1999. Tumor necrosis factor-alpha and basic fibroblast growth factor differentially inhibit the insulin-like growth factor-I induced expression of myogenin in $\mathrm{C} 2 \mathrm{C} 12$ myoblasts. Exp. Cell Res. 249:177-187.

23. Langen, R.C., Schols, A.M., Kelders, M.C., Wouters, 
E.F., and Janssen-Heininger, Y.M. 2001. Inflammatory cytokines inhibit myogenic differentiation through activation of nuclear factor-kappaB. FASEB J. 15:1169-1180.

24. Guttridge, D.C., Albanese, C., Reuther, J.Y., Pestell, R.G., and Baldwin, A.S., Jr. 1999. NF-kappaB controls cell growth and differentiation through transcriptional regulation of cyclin D1. Mol. Cell. Biol. 19:5785-5799.

25. Guttridge, D.C., Mayo, M.W., Madrid, L.V., Wang, C.-Y., and Baldwin, A.S., Jr. 2000. NF-KB-induced loss of MyoD messenger RNA: possible role in muscle decay and cachexia. Science. 289:2363-2366.

26. Moldawer, L.L., Svaninger, G., Gelin, J., and Lundholm, K.G. 1987. Interleukin 1 and tumor necrosis factor do not regulate protein balance in skeletal muscle. Am. J. Physiol. 253:C766-C773.

27. Spiegelman, B.M., and Hotamisligil, G.S. 1993. Through thick and thin: wasting, obesity, and TNF alpha. Cell. 73:625-627.

28. Argiles, J.M., and Lopez-Soriano, F.J. 1999. The role of cytokines in cancer cachexia. Med. Res. Rev 19:223-248.

29. Todorov, P., et al. 1996. Characterization of a cancer cachectic factor. Nature. 379:739-742.

30. Carbo, N., et al. 2000. Interleukin-15 antagonizes muscle protein waste in tumour-bearing rats. $\mathrm{Br} . \mathrm{J}$. Cancer. 83:526-531.

31. Emery, P.W., Lovell, L., and Rennie, M.J. 1984. Protein synthesis measured in vivo in muscle and liver of cachectic tumor-bearing mice. Cancer Res. 44:2779-2784.

32. Baracos, V.E., DeVivo, C., Hoyle, D.H., and Goldberg, A.L. 1995. Activation of the ATP-ubiquitinproteasome pathway in skeletal muscle of cachectic rats bearing a hepatoma. Am. J. Physiol. 268:E996-E1006.

33. Lecker, S.H., Solomon, V., Mitch, W.E., and Goldberg, A.L. 1999. Muscle protein breakdown and the critical role of the ubiquitin-proteasome pathway in normal and disease states. J. Nutr. 129(Suppl. 1):227S-237S.

34. Mitch, W.E., and Goldberg, A.L. 1996. Mechanisms of muscle wasting. The role of the ubiquitin-proteasome pathway. N. Engl. J. Med. 335:1897-1905.

35. Stitt, T.N., et al. 2004. The IGF-1/PI3K/Akt pathway prevents expression of muscle atrophy-induced ubiquitin ligases by inhibited FOXO transcription factors. Mol. Cell. 14:395-403.

36. Sandri, M., et al. 2004. Foxo transcription factors induce the atrophy-related ubiquitin ligase atrogin-1 and cause skeletal muscle atrophy. Cell. 117:399-412.

37. Clark, K.A., McElhinny, A.S., Beckerle, M.C., and Gregorio, C.C. 2002. Striated muscle cytoarchitecture: an intricate web of form and function. Annu. Rev. Cell Dev. Biol. 18:637-706.

38. Ladner, K.J., Caligiuri, M.A., and Guttridge, D.C. 2003. Tumor necrosis factor-regulated biphasic activation of NF-kappa B is required for cytokineinduced loss of skeletal muscle gene products. J. Biol. Chem. 278:2294-2303.

39. Ouaaz, F., and Beg, A.A. 1999. A critical role for the RelA subunit of nuclear factor $\mathrm{kB}$ in regulation of multiple immune-response genes and in Fasinduced cell death. J. Exp. Med. 189:999-1004.

40. Swoap, S.J. 1998. In vivo analysis of the myosin heavy chain IIB promoter region. Am. J. Physiol. 274:C681-C687.

41. Wheeler, M.T., Snyder, E.C., Patterson, M.N., and Swoap, S.J. 1999. An E-box within the MHC IIB gene is bound by MyoD and is required for gene expression in fast muscle. Am. J. Physiol. 276:C1069-C1078.

42. Davis, R.L., Cheng, P.F., Lassar, A.B., and Weintraub, H. 1990. The MyoD DNA binding domain contains a recognition code for muscle-specific gene activation. Cell. 60:733-746.

43. Strassmann, G., Fong, M., Kenney, J.S., and Jacob, C.O. 1992. Evidence for the involvement of interleukin 6 in experimental cancer cachexia. J. Clin. Invest. 89:1681-1684.

44. Strassmann, G., Masui, Y., Chizzonite, R., and Fong, M. 1993. Mechanisms of experimental cancer cachexia. Local involvement of IL-1 in colon-26 tumor. J. Immunol. 150:2341-2345.

45. Yasumoto, K., et al. 1995. Molecular analysis of the cytokine network involved in cachexia in colon 26 adenocarcinoma-bearing mice. Cancer Res. 55:921-927.

46. Strassmann, G., et al. 1993. Suramin interferes with interleukin- 6 receptor binding in vitro and inhibits colon-26-mediated experimental cancer cachexia in vivo. J. Clin. Invest. 92:2152-2159.

47. Mendell, J.R., and Engel, W.K. 1971. The fine structure of type II muscle fiber atrophy. Neurology. 21:358-365.

48. Diffee, G.M., Kalfas, K., Al-Majid, S., and McCarthy, D.O. 2002. Altered expression of skeletal muscle myosin isoforms in cancer cachexia. Am. J. Physiol. 283:C1376-C1382.

49. Goldberg, A.L. 2000. Probing the proteasome path- way. Nat. Biotechnol. 18:538-543.

50. Temparis, S., et al. 1994. Increased ATP-ubiquitindependent proteolysis in skeletal muscles of tumor-bearing rats. Cancer Res. 54:5568-5573.

51. Medina, R., Wing, S.S., and Goldberg, A.L. 1995. Increase in levels of polyubiquitin and proteasome mRNA in skeletal muscle during starvation and denervation atrophy. Biochem. J. 307:631-637.

52. Lecker, S.H., et al. 2004. Multiple types of skeletal muscle atrophy involve a common program of changes in gene expression. FASEB J. 18:39-51.

53. Todorov, P.T., Field, W.N., and Tisdale, M.J. 1999. Role of a proteolysis-inducing factor (PIF) in cachexia induced by a human melanoma (G361). Br. J. Cancer. 80:1734-1737.

54. Acakpo-Satchivi, L.J., et al. 1997. Growth and muscle defects in mice lacking adult myosin heavy chain genes. J. Cell Biol. 139:1219-1229.

55. Garcia-Martinez, C., Costelli, P., Lopez-Soriano, F.J., and Argiles, J.M. 1997. Is TNF really involved in cachexia? Cancer Invest. 15:47-54.

56. Li, Y.P., and Reid, M.B. 2000. NF-kappaB mediates the protein loss induced by TNF-alpha in differentiated skeletal muscle myotubes. Am. J. Physiol. 279:R1165-R1170

57. Li, Y.P., et al. 2003. TNF-alpha increases ubiquitinconjugating activity in skeletal muscle by up-regulating UbcH2/E220k. FASEB J. 17:1048-1057.

58. Fong, Y., et al. 1989. Cachectin/TNF or IL-1 alpha induces cachexia with redistribution of body proteins. Am. J. Physiol. 256:R659-R665.

59. Kaplan, E.H., et al. 1990. Phase II study of recombinant human interferon gamma for treatment of cutaneous T-cell lymphoma. J. Natl. Cancer Inst. 82:208-212.

60. Krigel, R.L., et al. 1988. A phase I study of recombinant interleukin 2 plus recombinant beta-interferon. Cancer Res. 48:3875-3881.

61. Li, Y.P., Schwartz, R.J., Waddell, I.D., Holloway, B.R., and Reid, M.B. 1998. Skeletal muscle myocytes undergo protein loss and reactive oxygen-mediated NF-kappaB activation in response to tumor necrosis factor alpha. FASEB J. 12:871-880

62. Solomon, V., and Goldberg, A.L. 1996. Importance of the ATP-ubiquitin-proteasome pathway in the degradation of soluble and myofibrillar proteins in rabbit muscle extracts. J. Biol. Chem. 271:26690-26697.

63. Williams, A.B., et al. 1999. Sepsis stimulates release of myofilaments in skeletal muscle by a calciumdependent mechanism. FASEB J. 13:1435-1443. 